\title{
Structure of the cuticle of some ptyctimine oribatids (Acari: Oribatida)
}

\author{
JAROSLAV SMRŽ
}

Department of Zoology, Charles University, Viničná 7, Prague 2, CZ-128 44, Czech Republic; e-mail: smrz@mbox.cesnet.cz

Key words. Cuticular layers, cerotegument, light microscopy, TEM, Acari, Oribatida, Euphthiracaroidea, Phthiracaroidea

\begin{abstract}
The cuticle of Rhysotritia duplicita (Euphtiracaroidea: Euphtiracaridae), Phthiracarus sp., Steganacarus magnus, S. striculus and Tropacarus carinatus (all Phthiracaroidea: Phthiracaridae) was studied by light and transmission electron microscopy. A combination of light and electron microscopy were used to precisely characterize and classify the cuticular layers in oribatid mites and show how they differe in mites and other arthropods. In Rhysotritia duplicata the cuticle of the aspis (upper shield of the prosoma) differs significantly from that of the opisthosoma. Moreover, prosomal cuticle in this species is different from that of all others species studied. There were no structural differences in the cuticle of the prosoma and opisthosoma of species of the superfamily Phthiracaroidea (Phthiracarus, Steganacarus and Tropacarus) in spite of the differences in external apparence. Moreover, in Tropacarus, the rough sculpturing of the cuticle results from the very rough exocuticle, which is unlike the thick smooth cerotegument of the cuticle in Steganacarus.
\end{abstract}

\section{INTRODUCTION}

The structure of the cuticular layers of mites has been studied by several authors (Oribatida: Michael, 1884; Grandjean, 1951; Woodring \& Cook, 1962; Tarba \& Semenova, 1976; Pugh et al., 1987; Smrž, 1989; Iordansky \& Stein-Margolina, 1993; Ixodida: Balashov, 1979; Hackman, 1982). General texbooks on acarology have summarized the facts (Krantz, 1978; Evans, 1992). The most thorough study by Alberti et al. (1981) compared the cuticular ultrastructure of most of the important mite groups. Differences were revealed in the cerotegument and the structure of cuticular layers. The general internal microanatomy and ultrastructure of mites, including the cuticular structure, is described and discussed in the textbook by Alberti \& Coons (1999).

Several integumental layers can be distinguished under the light microscope when Masson's triple stain is used (Smrž, 1989, 1992, 1994):

Cerotegument - rough lipoid (Krantz, 1978) layer on the cuticular surface, which has frequently incorporated in it material from the environment (mineral or litter particles). It can be separated or removed from the cuticle, e.g., by dissolving it in lactic acid or simple abrasion. Some types of cerotegument are destroyed during the embedding process for histological sectioning. Cerotegument is not stained by Masson's triple stain.

Epicuticle - thin uppermost cuticular layer, hyaline, translucent, without any internal division or structure when viewed under a light microscope. It is not stained by Masson's triple stain, but easily to distinguished from the other layers.

The underlying layer can be homogeneous or divided into several sublayers. These can be termed exocuticle and endocuticle following the usual arthropod cuticular terminology or, together, as procuticle. But some mites exhibit several types of "exocuticle" or "endocuticle", which cannot be homologized with the exocuticle or endocuticle of other arthropods. This is a common feature in mites. This phenomenon is discussed by Alberti (Alberti et al., 1981; Alberti \& Coons, 1999). He proposed the more general term "layers of procuticle" based on the very rich and diversified scale of types of cuticles studied. The structural diversity is better described by this terminology. In this paper, the naming of the layers and sublayers is sensu Alberti (Alberti et al., 1981; Alberti \& Coons, 1999), with similarity to the conventional, arthropodological terminology indicated in brackets for comparison. The main procuticular sublayers are those previously observed in oribatids using light microscopy after staining with Masson's triple stain (Smrž, 1989, 1992, 1994, 1995):

Exocuticle - amber-coloured layer, not stained by Masson's triple stain. It has no internal structure or lamellae or striae perpendicular to the cuticle surface.

Endocuticle - without any internal structure, very conspicuous carmine colour when stained with Masson's triple stain.

The flexible cuticle between body parts and leg segments is of endocuticular nature and continuous with that of the neighbouring hard cuticular parts. It stains carmine red.

As mentioned below, there are several other types of layers.

Of course, TEM reveals more details. Such observations support the structures described above, although the interpretation of the striation or lamellar structures differs. Moreover, the cerotegument is better preserved in TEM than in histological preparations. The layers with different electron densities, however, correspond with those revealed by Masson staining. Differences between these two methods may also result from the different processes of specimen preparation (e.g. fixative used). As mentioned above, these studies supported the more general terminology - procuticle sublayers. 
The ptyctimine oribatids [the cohort Ptyctimina sensu Krantz (1978)] have a unique body division. Their prosoma is capable of ptychoidy, i.e. it is able to close on its opisthosoma like the blade of a knife [therefore "jackknife" mites: Krantz (1978)]. The dorsal shield of the prosoma - aspis - protects the appendages of the prosoma (Fig. 1). Zelenková (1986) records differences in the structure of cuticle of prosoma and opisthosoma of $R h y$ sotritia duplicata, as did Štambergová (2000).

I use the terms exocuticle 1, 2 or 3 and endocuticle 1 for the procuticular layers (exo- and endocuticle). These terms denote procuticular layers similar, but different from the traditional exocuticle and endocuticle. They do not describe the sequences of layers in the procuticle.

This study compares cuticular structure and ultrastructure of several members of two superfamilies of ptyctimine oribatids - Euphthiracaroidea and Phthiracaroidea.

\section{MATERIAL AND METHODS}

Rhysotritia duplicata (Grandjean, 1953), Phthiracarus sp., Steganacarus striculus (C.L. Koch, 1836), Steganacarus magnus (Nicolet, 1855) and Tropacarus carinatus (C.L. Koch, 1841) were extracted from soil symplex using Berlese-Tullgren funnels collected on moist filter paper and subsequently fixed and prepared for microscopic studies:

Specimens for light microscopy (LM) were fixed in BouinDubosque-Brasil fluid modified for oribatids (Smrž, 1989), embedded in Histoplast (Serva), sectioned using a MSE rotation microtome (thickness $5 \mu \mathrm{m}$ ), stained with Masson's triple stain, observed under a Provis AX 70 microscope (Olympus) and the images edited using the Microimage 3.1 image analysis programme (Olympus). The application of Nomarski DIC and inversion of the image in image analysis is very useful.

Specimens were fixed for TEM in cacodylate-buffered glutaraldehyde (4\%), postfixed in $1 \%$ osmium tetroxide, embedded in Spurr medium and sectioned using an Ultracut ultramicrotome (Reichert). Sections were stained in lead citrate and uranyl acetate and observed under a Philips EM 300 transmission electron microscope.

\section{RESULTS}

\section{Rhysotritia duplicata (Figs 1-7)}

The prosomal cuticle consists of a very thin epicuticle and procuticle of three sublayers: two thick lamellar sublayers ("exocuticle 1" and "2") and a thinner sublayer ("endocuticle") (Figs 2, 3). TEM confirmed the existence of two sublayers of "exocuticle". The upper sublayer consists of parallel smooth lamellae (Fig. 4), whereas the underlying sublayer has a more complicated structure (Fig. 5). In the opisthosomal region, however, the cuticle consists of an epicuticle, above a relatively thin, greenish stained, finely striated and lamellar procuticular layer ("exocuticle 3"), which is underlain by a green stained (Masson) hyaline layer similar to the binding tissue or, maybe, to the endocuticle-like layer ("endocuticle 1"). However, this is stained differently from the standard(Fig. 6). TEM revealed a relatively thick epicuticle above a lamellar "exocuticle 3", which is underlain by a thin nonstructured layer ("endocuticle 1"). Therefore, we can compare the prosomal with the opisthosomal cuticle (Figs 2 and 3 with 6 ). The leg cuticle has the usual structure: epicuticle and with an amber coloured "exocuticle" and conspicuously red stained "endocuticle". There are conspicuous large cells beneath the cuticle of the legs (Fig. 7). The same structure was found in other species.

\section{Steganacarus striculus (Figs 8-9)}

Both body parts are covered by the same type of cuticle consisting of a conspicuous epicuticle and procuticle composed of an amber coloured striated ("exocuticle 3") and thin red coloured ("endocuticle") sublayers (Figs 8, 9).

\section{Steganacarus magnus (Figs 10-12)}

The cuticular structure of the body and legs resembles that of previous species. The cuticle has superficial small pits covered by a rough cerotegument, which is easily separated from the cuticle surface (Fig. 11). Because of the cerotegument the rough nature of the body surface is more conspicuous (Fig. 10). TEM revealed very fine lamellae running parallel to the surface and allochthonous particles, of inorganicand organic origin in the cerotegument (Fig. 12).

\section{Phthiracarus sp.}

The same type of cuticle covers both part sof the body and resembles that of $S$. striculus. It consists of a conspicuous epicuticle and procuticle composed of amber coloured striated "exocuticle 3" and a thin red coloured "endocuticle" sublayers.

\section{Tropacarus carinatus (Figs 13-14)}

The composition of the prosomal and opisthosomal cuticle is similar to that of the previous species, but the upper procuticular sublayer ("exocuticle 3") is conspicuously thick, the underlying one ("endocuticle") relatively very thin. Moreover, "exocuticle 3" is remarkably shaped into high ridges, rather "waves", on its upper surface. They cover the whole body (Fig. 14) forming the characteristic pits on the body surface (Fig. 13).

\section{DISCUSSION}

As mentioned above, the terminology of Alberti (Alberti et al., 1981, Alberti \& Coons, 1999) is suitable for the describing variability in oribatid (maybe, all mites) cuticle. On the other hand, some features (striation, staining in LM, lamellae or lamellar parabolic fibres in TEM) appear to be characteristic of some layers. The exocuticle sublayers are very diverse and better differentiated by the terminology "exocuticle 1, 2, 3". Therefore, both types of terminology are used in this paper for orientation and comparison. The traditional, strict division of cuticle into exo- and endocuticle (cf. insect cuticle: Imms, 1973 ) is insufficient. There are more sublayers of procuticle, which are often very similar to each other (cf. Evans, 1992; Alberti \& Coons 1999).

The two superfamilies of ptyctimine oribatids have noticeably different cuticle structures. In the superfamily Euphthiracaroidea, there are two different types of "exocuticle" (or, better, upper procuticle sublayers) on the prosoma and opisthosoma. This supports the assumption of Zelenková (1986) and Štambergová (2000) (see Intro- 


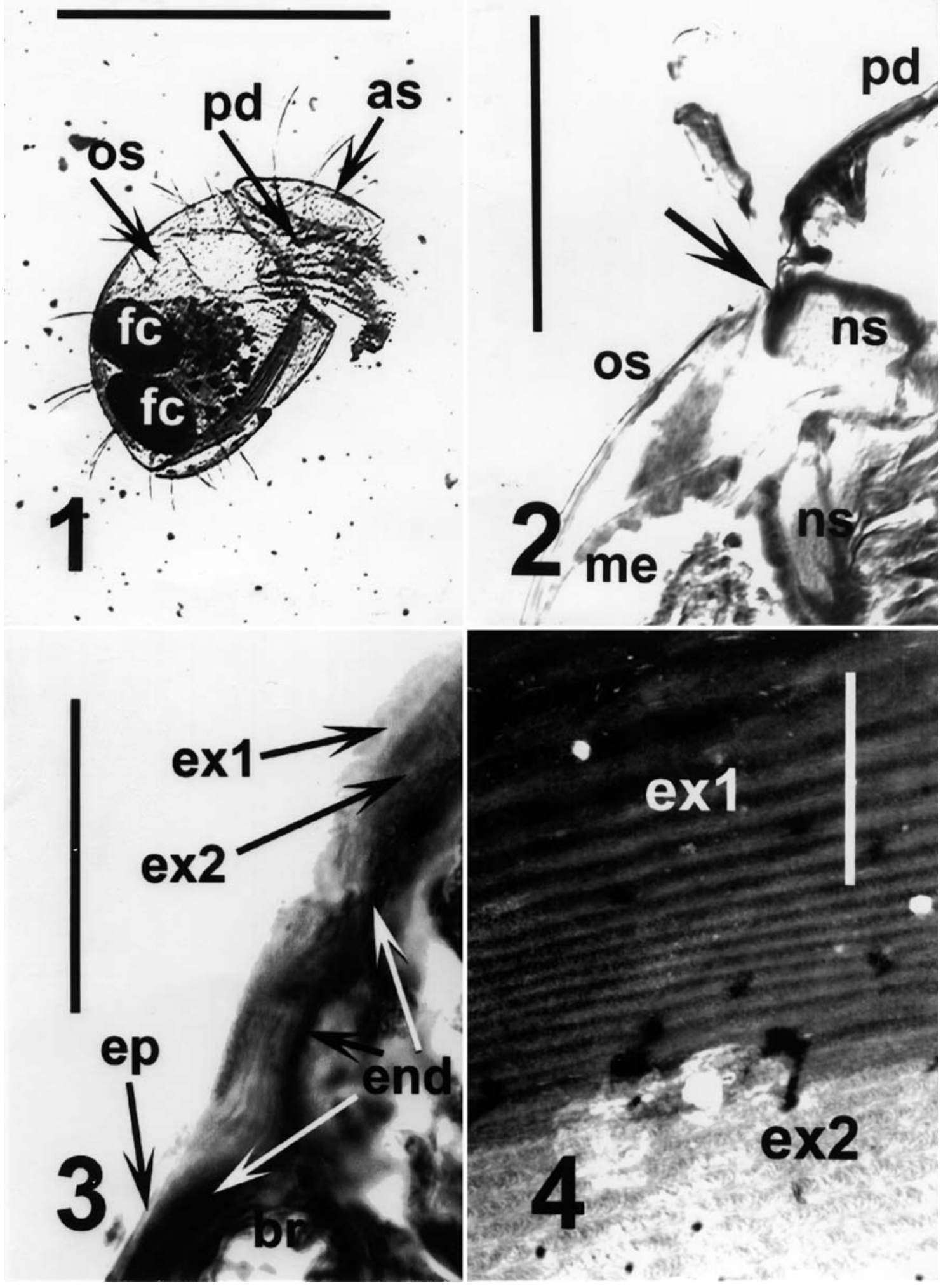

Figs 1-4: Rhysotritia duplicata. 1 - intact specimen, female, lateral view; 2 - sagittal section through the prosoma-opisthosoma junction, note the cuticular differences between body parts; 3 - details of the cuticle of the prosoma with a thick three-layered procuticle, sagittal section; 4 - details of the prosomal cuticle, two sublayers of the exocuticular nature, TEM, sagittal section. Intact specimen in lactic acid (1), Masson's triple stain (2-3), TEM (4). Scales: $300 \mu \mathrm{m}$ (1), $20 \mu \mathrm{m}$ (2), $5 \mu \mathrm{m}$ (3), $0.5 \mu \mathrm{m}$ (4). Abbreviations used: as - aspis, br - bothridium, end - endocuticle, ep - epicuticle, ex1, ex 2 - two procuticular sublayers of exocuticular nature, which are different from the standard exocuticle (cf. ex in the Fig. 7), fc - faecal pellet, me - mesenteron, ns - central nervous synganglion, os - opisthosoma, pd - prosoma. 


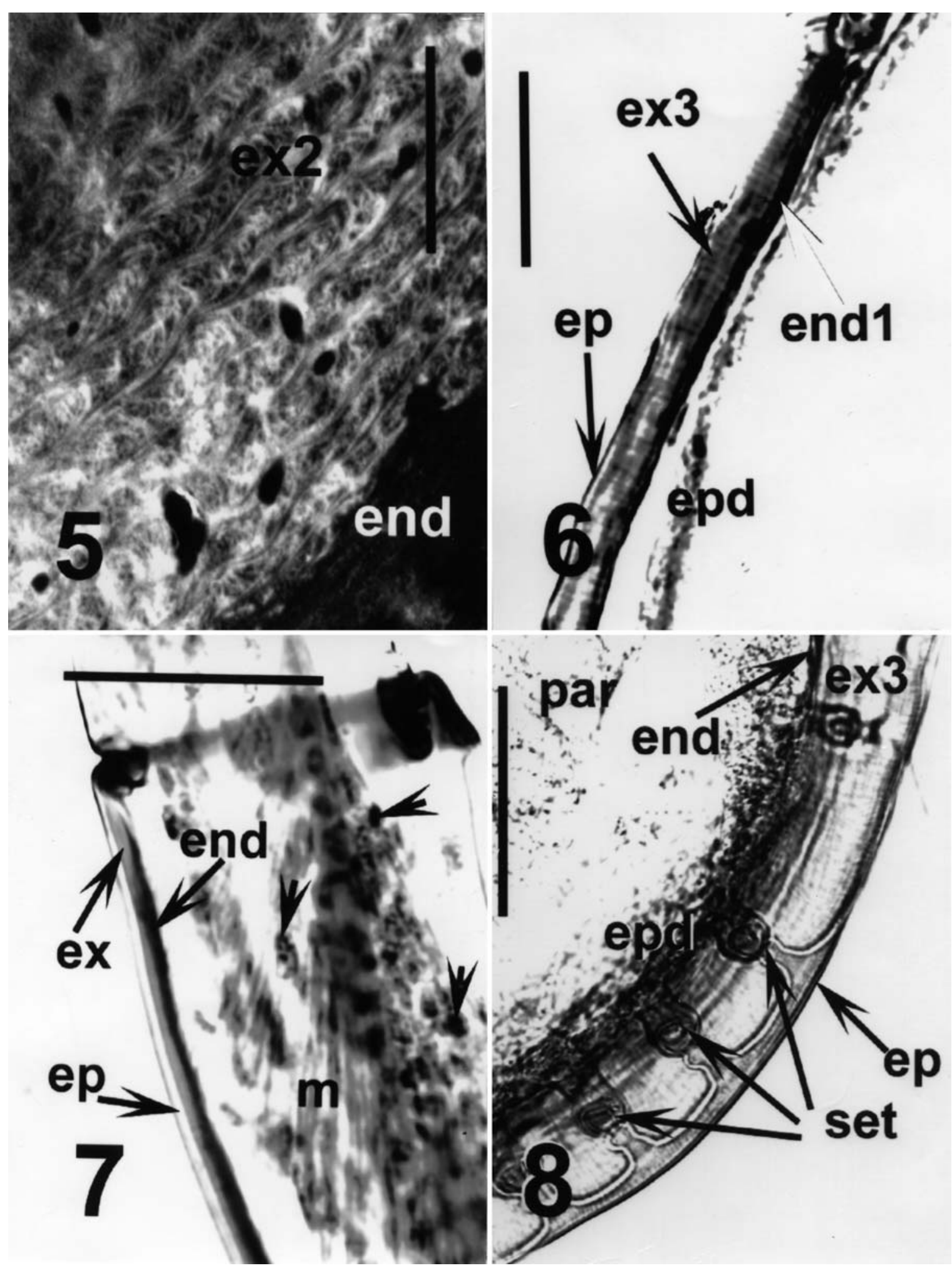

Figs 5-8. 5-7: Rhysotritia duplicata, sagittal sections. 5 - the second prosomal procuticular sublayer of exocuticular nature (ex 2 cf. ex1 in the Fig. 4), TEM; 6 - the opisthosomal cuticle differs from that of the prosoma, (cf. Figs 2, 3), Nomarski DIC; 7 - leg, generally its cuticle differs from that of the body type, arrowheads indicate the free cells of oenocyte type; 8 - Steganacarus striculus - anal valves with same type of cuticle as the body (see ex3), sagittal section, Masson's triple stain $(6,7,8)$, TEM (5). Scales: $10 \mu \mathrm{m}(6,7), 5 \mu \mathrm{m}(8), 0.5 \mu \mathrm{m}(5)$. Abbreviations used: end - procuticular sublayer of the standard endocuticular nature, end 1 - procuticular sublayer of endocuticular nature, different from standard endocuticle (cf. "end" in Figs 5, 7), ep - epicuticle, epd epidermis, ex - the procuticular sublayer of standard exucuticular type, ex2, ex 3 - procuticular sublayers, different from standard exocuticle (cf."ex" in Fig. 7), m - muscles, par - mesenchyma, set - insertion of setae. 

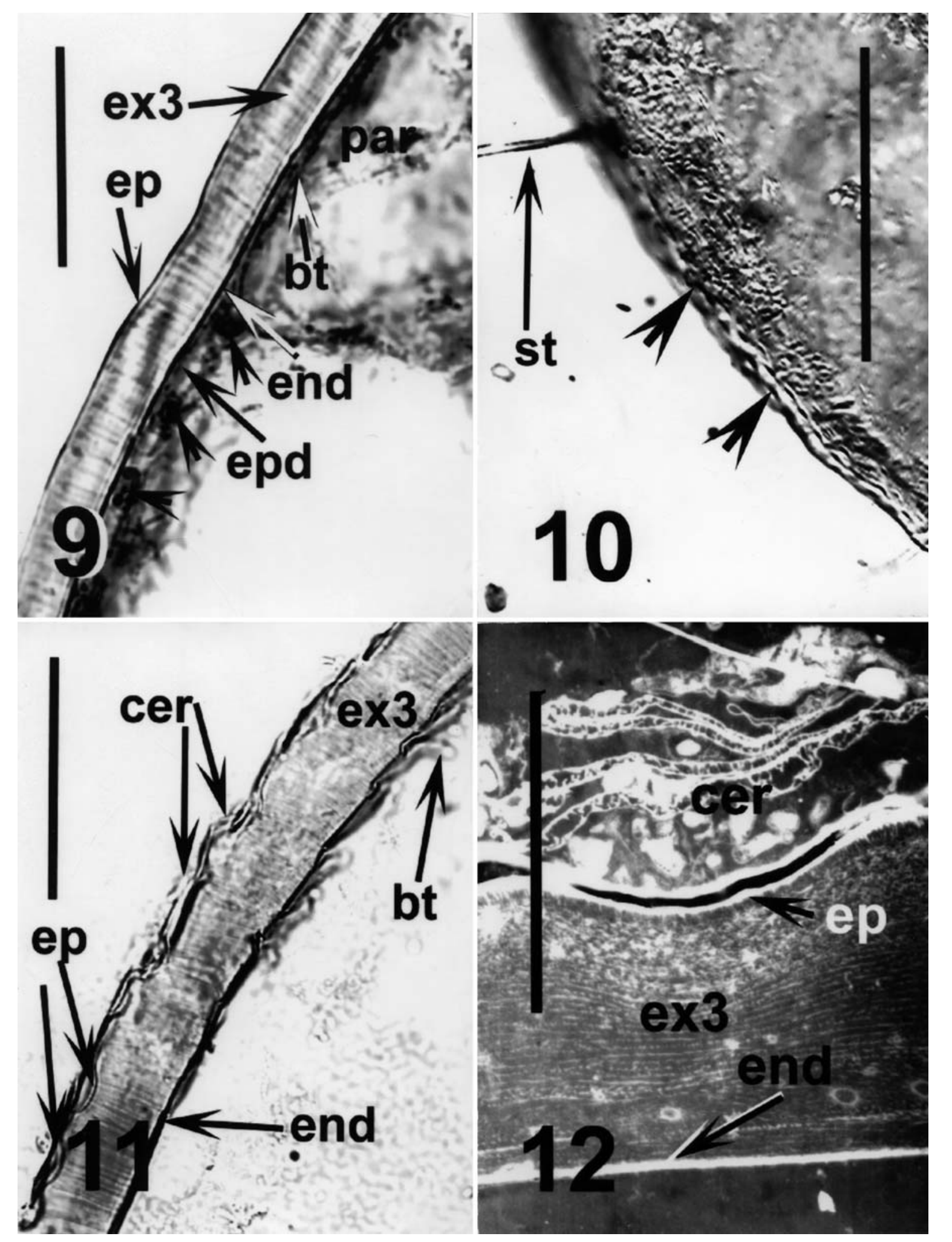

Figs 9-12: Steganacarus. 9 - S. striculus, dorsal opisthosomal (notogastral) cuticle, with "ex3", small arrowheads indicate epidermal nuclei, sagittal section; 10 - S. magnus surface of dorsal opisthosoma, intact specimen, Nomarski DIC, arrowheads indicate the surface sculpture (pits); 11 - the same species, dorsal opisthosomal cuticle, with "ex3", sagittal section; 12 - the same species, dorsal opisthosomal cuticle overlain by cerotegument with allochthonous particles (mineral particles and probably fungal hyphae), sagittal section, TEM. Intact specimen in lactic acid (10), Masson's triple stain (9, 11), TEM (12). Scales: $20 \mu \mathrm{m}(10), 5 \mu \mathrm{m}(9,11)$, $2 \mu \mathrm{m}$ (12). Abbreviations used: bt - binding tissue, cer - cerotegument, end - procuticular sublayer of the standard endocuticular nature, ep epicuticle, epd - epidermis, ex3 - procuticular sublayer "exocuticle 3" different from the standard exocuticle, par - parenchyma, st - notogastral seta. 


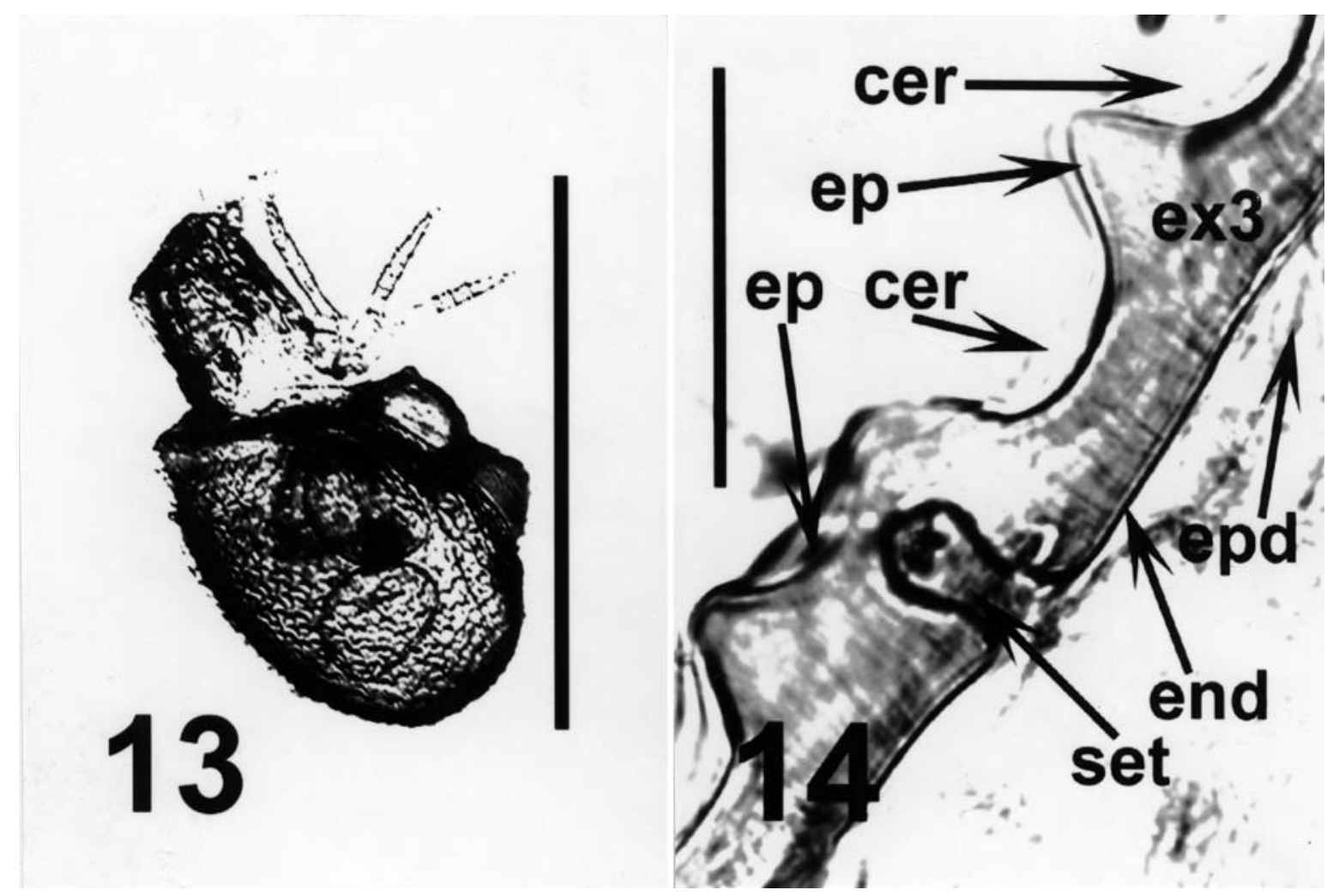

Figs 13-14. 13 - Tropacarus carinatus - intact specimen, lateral view; 14 - the same species, details of the opisthosomal cuticle with "ex3" (see above), Nomarski DIC, sagittal section. Nomarski DIC (13), Masson's triple stain (14). Scales: $400 \mu \mathrm{m}(13), 5 \mu \mathrm{m}$ (14). Abbreviations used: cer - cerotegument, end - procuticular sublayer of standard endocuticular nature, ep - epicuticle, epd epidermis, ex3 - procuticular sublayer "exocuticle 3", different from standard exocuticle (cf."ex" in Fig. 7), set - insertion of notogastral seta.

duction). Moreover, the opisthosoma of Rhysotritia lacks the usual procuticular sublayer of an endocuticular nature. The examination of intact specimens of Rhysotritia did not reveal conspicuous differences between the cuticle of the prosoma and opisthosoma, although the heavier sclerotization of the aspis (prosomal dorsal shield) is visible. TEM revealed some features of the prosomal cuticle - three subepicuticular layers (two sublayers of "exocuticle" or simply two procuticular sublayers, and a third - of an endocuticular nature or position). The lamellar upper sublayer is underlain by a sublayer of parabolic pattern (cf. gamasid Veigaia: Alberti \& Coons, 1999). In Rhysotritia, the peculiarly strengthened structure of both sublayers supports the structural and functional specialization of the prosoma. For moving through soil the prosomal shield - aspis - needs to be resistant to abrasion (cf. Dinsdale, 1974). On the other hand, the cuticle of the very large opisthosoma can be thinner as it only covers of the internal organs. In comparison to other ptyctimine mites (Steganacarus, Phthiracarus, Tropacarus), there are conspicuous differences in the prosoma and opisthosoma in intact and the sectioned Rhysotritia mites.

The cuticle of phthiracaroid mites has the same structure on both body parts, although their surfaces differ in intact mites:

a) nearly smooth in Phthiracarus sp. and Steganacarus striculus b) rough in Steganacarus magnus and Tropacarus carinatus.

The internal structure, however, seems to be similar in all studied species. In LM, the perpendicular striation is clear. The parallel lamellae, however, are conspicuous only in TEM. On the other hand, there are some differences in the role of cerotegument and cuticle in the sculpturing of the body surface in the latter group: the cerotegument gives rise to the rough superficial sculpture in Steganacarus magnus, whereas in Tropacarus carinatus it is the exocuticle.

The leg cuticle of studied species has of the same structure, probably because it has the same major function insertion of muscles. In unfavourable conditions, ptyctimine oribatids, can retract thein legs under thein aspis. Hence, protective modifications of cuticle or cerotegument appear to be redundant. An enormous number of free cells was prezent in the legs (oenocytes sensu Smrž, 1995; cf. Romer \& Gnatzy, 1981) of all species studied.

Differences between the two superfamilies are clear. Maybe, they result from phylogeny, or the different life patterns of the species (cf. Strenzke, 1952; Dinsdale, 1974; Weigman \& Kratz, 1981) and resistance requirements (Madge, 1964).

The methods used for cuticle analysis were complementary. The main differences were in the definition of striae (perpendicular to the body surface) and lamellae (parallel to the body surface). The former structures were 
clearly visible in LM, and the latter in TEM. These differences may have resulted from the different wals in which the specimen were prapared. TEM revealed the very fine details necessary for accurate descriptions, but Masson's triple stain distinguished the main characteristics and useful details after preparation of the specimens for examination under high magnification followed by image analysis. Therefore, their combination provides a useful method for the clasification of cuticular structures.

ACKNOWLEDGEMENTS. I am very obliged to M. Luxton, National Museum of Wales, Cardiff, for his helpful linguistic, stylistic and scientific review of this paper. I am grateful to G. Alberti, University of Greifswald for his very valuable advice on oribatid cuticle and interpretation of micrographs. I wish to thank M. Doubek, State Health Institute, Prague, for his very kind help with the TEM observations, J. Vávra, Department of Parasitology, Charles University, Prague, who rendered possible to make the ultramicrotome sections. This work was funded by grant GAČR 526/02/0681 - laboratory study, and a grant from Ministry of Education of Czech Republic 21-3130046 - field study.

\section{REFERENCES}

Alberti G. \& Coons L.B. 1999: Acari: Mites. In Harrison F.W \& Foelix R.F (eds): Microscopic Anatomy of Invertebrates. Vol. AC. Chelicerate Arthropoda. Wiley \& Sons, Chichester, pp. 515-1215.

Alberti G., Storch V. \& RenNer H. 1981: Über den feinstrukturellen Aufbau der Milbencuticula (Acari, Arachnida). Zool. Jb. Anat. 105: 183-236.

Balashov Y.S. 1979: Atlas of Electronmicroscopical Anatomy of Ticks. Nauka, Leningrad, 256 pp. (in Russian).

Evans G.O. 1992: Principles of Acarology. CAB International, Walingford, $536 \mathrm{pp}$.

DinsDAle D. 1974: Feeding activity of a phthiracarid mite (Arachnida: Acari). J. Zool. Soc. London 174: 15-21.

Grandjean F. 1951: Sur le tegument des Oribates (2-serie). Bull. Mus. Natl. Hist. Nat. 23: 497-507.

Hackman R.H. 1982: Structure and function in tick cuticle. Annu. Rev. Entomol. 27: 75-95.

Imms A.D. 1973: A General Textbook of Entomology. 9th ed. Chapman and Hall, London, 886 pp.

IORDANSKY S.N. \& SteIn-Margolina V.A. 1993: The cuticle structure and xeroresistance of the deutonymph and adult oribatid mite Tectocepheus velatus (Acariformes, Oribatei). Zool. Zh. 72: 30-42 (in Russian).

Krantz G.W. 1978: A Manual of Acarology. 2nd ed. Oregon State University, Corvallis, 509 pp.

Madge D. 1964: The water relations of Belba geniculosa Oudemans, and other species of Oribatid mites. Acarologia 6: 199-223.

Michael A.D. 1884: British Oribatidae. Vol. 1. Ray Society, London, $333 \mathrm{pp}$.

Pugh P.J.A., King P.E. \& Fordy M.R. 1987: A comparison of the structure and function of the cerotegument in two species of Cryptostigmata (Acarina). J. Nat. Hist. 21: 603-616.

Romer F. \& GNATZy W. 1981: Arachnid oenocytes: ecdysone synthesis in the legs of harvestmen (Opilionidae). Cell Tissue Res. 216: 449-453.

SMRž J. 1989: Internal anatomy of Hypochthonius rufulus (Acari: Oribatida). J. Morphol. 200: 215-230.

SMRž J. 1992: Some adaptive features in the microanatomy of moss-dwelling oribatid mites (Acari: Oribatida) with respect to their ontogenetical development. Pedobiologia 36: 306-320.

SMRž J. 1994: Survival of Scutovertex minutus (Koch) (Acari: Oribatida) under differing humidity conditions. Pedobiologia 38: $448-454$

SMRž J. 1995: Free cells in the body cavity of oribatid mites (Acari: Oribatida). Pedobiologia 39: 488-495.

ŠTAMBERGOVÁ M. 2000: Moss mites (Acari, Oribatida) in the soils in the gardening and adjoining plots in Hradec Králové city. Thesis, Charles University, Prague, 75 pp. (in Czech)

StrenzKe K. 1952: Die Oribatiden und ihre Synusien in den Böden Norddeutschland. Zoologica (Stuttgart) 172 pp.

TARBa Y.M. \& Semenova L.M. 1976: Cuticle structure of the Oribatei in relation to their ecology. Pedobiologia 16: 127-135.

WeIGMANn G. \& KRAtZ W. 1981: Die deutschen Hormilbenarten und ihre ökologische Charakteristik. Zool. Beitr. 27: 459-489.

Woodring J.P. \& CooK E.F. 1962: The internal anatomy, reproductive physiology, and molting process of Ceratozetes cisalpinus (Acarina: Oribatei). Ann. Entomol. Soc. Am. 55: 164-181.

Zelenková M. 1986: Anatomy, biology and ecology of Rhysotritia duplicata (Grandjean, 1953) (Acarina, Oribatida, Euphthiracaridae). Thesis, Charles University, Prague, 102 pp.

Received September 30, 2003; revised March 4, 2004; accepted April 13, 2004 